\title{
Social Stimuli, Testosterone, and Aggression in Gull Chicks: Support for the Challenge Hypothesis
}

\author{
Albert F. H. Ros,* Steph J . Dieleman, $†$ and \\ Ton G. G. Groothuisł, ${ }^{1}$ \\ *Eco- and Ethology Research Group, Instituto Superior de Psicologia Aplicada, Rua Jardim \\ do Tabaco 34, 1149-041 Lisbon, Portugal; +Department of Herd Health and Reproduction, \\ University of Utrecht, Yalelaan 7, 3584 CL, Utrecht, The Netherlands; and $\ddagger$ Department of \\ Animal Behaviour, Biological Centre, University of Groningen, Kerklaan 30, \\ 9750 AA Haren, The Netherlands
}

Received August 10, 2001; revised November 20, 2001; accepted November 21, 2001

\begin{abstract}
We tested the challenge hypothesis for the hormonal regulation of aggression in chicks of the black-headed gull, Larus ridibundus. Chicks of this species are highly aggressive toward conspecifics, but never to peers that hatched from the same clutch (modal clutch size is three). Therefore, in the first experiment small families were housed together in large groups (challenged condition) and compared to families kept isolated (nonchallenged condition). As expected, in the challenged condition during the initial stage of territory establishment basal levels of testosterone $(T)$ were clearly higher than those in the nonchallenged condition. In the second experiment we tested the effect of a short social challenge on short-term T-fluctuations. The design was based on an earlier experiment, showing that after temporary T-treatment chicks become very sensitive to social challenges while having low basal T-levels. We now show that these social challenges induce brief elevations in plasma T-levels. These peaks are similar to those in previously untreated chicks but untreated chicks do not respond with aggression to a challenge. Therefore, we conclude that the initial exposure to elevated T-levels increases the sensitivity to brief changes in $T$ induced by social challenges. In this way exposure to $T$, that may be detrimental for development, is minimized while birds remain able to defend territories. This is the first report showing that the challenge hypothesis as established for adult birds, is also applicable for aggressive behavior in young birds outside the sexual context. Furthermore we suggest that a phase of priming with $T$ is necessary to obtain the high behavioral responsiveness to a challenge. ๑ 2002 Elsevier Science (USA)
\end{abstract}

\footnotetext{
${ }^{1}$ To whom correspondence and reprint requests should be addressed. E-mail: T.Groothuis@biol.rug.nl.
}

Key Words: testosterone; challenge hypothesis; aggression; black-headed gull; territorial behavior; chick; ontogeny; priming; sensitization; organizing effects; external stimuli.

Testosterone is considered to have a function in preparing the animal for a high level of social competition. In birds, testosterone facilitates aggressive behavior and increases muscle growth, whereas it inhibits parental care, fat deposition and molt (reviewed in Ketterson, Nolan, Cawthorn, Parker, and Ziegenfus, 1996). Although all these effects may play an important role in the optimization of social competitiveness, some of these effects are detrimental to the animal under chronic exposure (Dufty, 1989; Moss, Parr, and Lambin, 1994; Ketterson et al., 1996). Consequently, testosterone production should be tuned to the period in which the animal faces important social challenges. Indeed the pattern of testosterone secretion in the course of the season seems to be related to the type of social system of a species (Wingfield, Hegner, Dufty, and Ball, 1990). In polygamous species, males are generally not involved in parental care, face high competition throughout the breeding season, and maintain high basal levels of testosterone. In monogamous species, high basal levels of testosterone are present only during a restricted period when territorial establishment and mate selection take place. Basal levels of testosterone are low when parental care is provided although males still respond aggressively to intruders at the territory. Several studies demonstrate that this 
aggressive responsiveness to a challenge is facilitated by a rapid but only temporary release of testosterone (Harding, 1979; Ramenofsky, 1984; Wingfield, 1985; Hegner and Wingfield, 1987). The relationship between mating systems, social challenges, and hormone levels was synthesized in the challenge hypothesis (Wingfield et al., 1990).

So far the evidence in support of the challenge hypothesis comes exclusively from studies on adult birds in a sexual context. Interestingly, in several bird species young birds also perform aggressive behavior and do this in a nonsexual context (between sibling aggression: Cash and Evans, 1986; Mock, Lamey, Williams, and Pelletier, 1987; Drummond and Osorno, 1992; territorial aggression: Groothuis, 1989a and 1989b). Sibling aggression may not be regulated by testosterone (Nuñez de la Mora, Drummond, and Wingfield, 1996; Ramos-Fernandez, Nuñez de la Mora, Wingfield, and Drummond, 2000). However, there is convincing experimental evidence for testosterone-dependent regulation of territorial aggression in black-headed gull chicks (Groothuis and Meeuwissen, 1992). Young birds especially might be vulnerable to the subsequent effects of testosterone: like reduction of growth (Fennell and Scanes, 1992; Ros, 1999); modification of plumage characteristics (Ros, 1999); decrease of begging behavior; and modification of the syrinx (Groothuis and Meeuwissen, 1992). Therefore, if juvenile aggression is testosterone dependent, it is to be expected that also in these young testosterone profiles are finely tuned to the challenging situation.

In the black-headed gull modal clutch size is three, and chicks never show aggression toward their siblings. The chicks are however remarkably aggressive early in life toward intruders on the territory. They grow up in dense colonies in which they are completely dependent on the parents for food during the first 8 weeks after hatching. In the course of the first 2 weeks the chicks begin to demand so much food that often both parents have to leave the territory simultaneously to forage. Adult and juvenile birds that intrude on the territory to scavenge or to establish new territories then attack the chicks. The chicks respond to these challenges with vigorous performance of aggressive behavior (Groothuis, 1989b).

Similar to the field situation, aggression is lacking between the members of small (pseudo) family groups composed of not more than five chicks of the same age and reared together from the first week onward. However, high levels of aggression occur when such pseudo families are confronted with other families or with a model of an adult conspecific (Groothuis, 1989a, 1989b). It is not known whether such temporary increases of aggressive behavior are based on temporary changes in plasma levels of testosterone.

Groothuis and Meeuwissen (1992) studied the relation between testosterone and aggression in young black-headed gulls experimentally. They implanted chicks and juveniles with pellets of testosterone. This revealed that testosterone strongly promoted aggressive behavior in both age classes. After termination of the testosterone treatment basal levels of testosterone were low again but aggressive behavior was maintained at much higher levels than that of controls. Based on this the authors suggested that aggression had become independent of the hormone. However, brief elevations in testosterone levels during social challenges have been documented for adults of several species (the Challenge hypothesis: Wingfield et al., 1990), but these were not investigated in their study. Further, the temporal treatment may have increased the sensitivity of the birds to these peaks in testosterone levels later in life. Therefore, we studied whether the challenge hypothesis as postulated for adult birds, in particular the influence of social challenges on brief surges of testosterone, regulating aggression during periods of low basal levels of this hormone, can be applied to the regulatory mechanism of aggressive behavior in chicks. We studied also whether a temporary exposure to elevated basal levels of testosterone is needed to develop this regulation.

Two experiments with black-headed gull chicks were carried out. In the first experiment we analyzed whether exposure of the chicks to a period of territorial challenges, as is the normal situation in the field, is correlated with increased basal levels of testosterone. In the second experiment we used a similar set up as the one of Groothuis and Meeuwissen (1992). We analyzed (1) whether in the period when the experimentally elevated basal levels of testosterone were returned to low values, aggression is related to brief elevations of blood levels of the hormone around the time of a territorial challenge, and (2) whether this may be due to priming to testosterone in an earlier phase.

\section{METHODS}

\section{Design Experiment 1: Effect of Social Challenges on Basal T Levels}

Large group challenge. In order to test the effects of social stimulation on basal $\mathrm{T}$ levels we manipulated group size during early rearing. Seven chicks of 4 days 
old were housed in an indoor aviary. This group size is approximately twice as large as a normal sibling group in the field and pilot studies indicated that in such large groups many aggressive interactions occur. In the aviary, measuring $5 \times 1.5 \mathrm{~m}$, three nest boxes were placed for shelter. Blood samples were collected at day $5(N=5)$ and $18(N=3)$ after housing the chicks in one group in the aviary, and not contingent on social interactions.

The control group consisted of nonchallenged chicks, kept in small groups of 3 to 5 peers of similar ages. Since chicks in such small pseudo-sibling groups never fight with each other, these chicks were not exposed to aggressive challenges. Their sample size for each age class varied between 5 and 15 and is mentioned throughout the text.

Territorial challenge. In this setup we mimicked the territorial situation of the field. For this experiment we composed three pseudo-families (12 chicks; group size: three, four, and five chicks). They were each housed in one of three enclosures of $1 \times 1 \mathrm{~m}$. of the same indoor aviary of $1 \times 3 \mathrm{~m}$. The enclosures were separated with opaque partitions so that families could not see another family. Each family was provided with a nest box for shelter and was given 2 weeks to become territorial. At 2.5 weeks after hatching we removed the partitions between the families (challenged condition). Blood samples were collected at day $5,(N=12)$ and day $16,(N=12)$ after removal of the partitions, again not contingent on social interactions. The control group consisted of the nonchallenged chicks mentioned above, matched for the same age as this challenged group.

\section{Design Experiment 2: Priming by Temporary T- Implantation}

All families originally consisted of three chicks and were kept isolated from other families to prevent aggressive interactions. The experimental group comprised four families of which the chicks received testosterone implants 9 days after hatching (T group: $N=8$, group size 1-3 chicks: 4 chicks died because of respiratory problems, see below). We removed the implants after 10 days. The control group comprised of chicks that received sham-surgery ( $C$ group). For this, from each of five families we selected one chick. To assess the effectiveness of the implants plasma testosterone levels were analyzed in blood samples of the $\mathrm{T}$ group collected at 7 days after testosterone treatment. These were compared with those of the T and C group collected in the first week after termination of treatment: T group: $N=8, \mathrm{C}$ group: $N=5$. These samples were drawn in periods when no aggressive challenges occurred.

We assessed the effect of social challenges on aggressive behavior by simulating the context of an adult intruder on the territory. This was done by confronting the chicks with a stuffed adult conspecific (see below). These tests were carried out with 2-3 day intervals from the end of the implantation phase at 2.5 weeks after hatching until the period the chicks were able to fledge at 7 weeks. In order to assess the effect of exposure of the chicks to these aggressive challenges on levels of testosterone, blood samples were drawn within 5-10 min after behavioral testing. These were collected from the $\mathrm{T}$ and $\mathrm{C}$ group at the end of the fifth week ( $N=7, N=5$, respectively) and, in addition, from the $\mathrm{T}$ group at the end of the sixth week $(N=6)$.

\section{Rearing Conditions}

Eggs and chicks were randomly collected in the field at the age of 0-4 days after hatching, placed in small groups and hand reared. The small groups consisted of three to five peers to form pseudo-sibling families (here called families) mimicking the natural nest condition. Because sex differences in morphology develop only much later in life, sex of the birds was not established. It cannot be a confounding factor in the analyses because gulls show hardly any sexual differentiation in social behavior even in response to testosterone treatment (Terkel, Moore, and Beer, 1976; Wingfield, Newman, Hunt, and Farner, 1982; Groothuis and Meeuwissen, 1992). To facilitate individual recognition by the observer the chicks were individually marked on head or back with rhodamine or picrine (ICN Biochemicals, Cleveland, $\mathrm{OH}$; chemicals were dissolved in acetone) and received a color ring.

Except for the "Large Group Challenge" condition, we held the families in indoor cages measuring approximately $1 \times 1 \mathrm{~m}$, which is the average size of a territory in the field (Paterson, 1965); the floor was covered with straw. Families were visually isolated from each other. The chicks of the "Large Group Challenge" condition were housed in somewhat larger enclosures in larger groups. In the middle of each cage we positioned a 100-W lamp, which provided a temperature of approximately $37^{\circ} \mathrm{C}$. After 2 weeks the lamp was replaced by a 30-W lamp, which was removed 2 weeks later when the chicks were 1 month old. In addition, light was provided on the basis of $16 \mathrm{~h}$ light, $8 \mathrm{~h}$ dark schedule. Food and water were 
available ad libitum. During the first 2 weeks chicks were fed with a moistened mixture of food pellets for trout (Trouvit, Trouw, Gent, Belgium) and food for fowl chicks (Sivo start, Bogena, Waalwijk, The Netherlands). This basic diet was supplemented daily with smelt, Osmerus eperlanus, and mashed hard-boiled chicken eggs. At 2 weeks of age, the diet was gradually shifted to dry trout pellets, with mashed egg added twice a week. A vitamin supplement (Calviet, UTD, Meppel, The Netherlands) was added weekly.

After the experiments all birds were released in large outside aviaries. They have been used for other experiments in the course of the same or the next year. All experiments were carried out under specified Dutch governmental licenses.

\section{Testosterone Treatment and Blood Collection}

We administered testosterone, using silicon tubes (Medica BV, 's Hertogenbosch, The Netherlands; internal diameter: $1.0 \mathrm{~mm}$, external diameter: $3.0 \mathrm{~mm}$, length of column: $12 \mathrm{~mm}$, closed on both ends with 1 $\mathrm{mm}$ silicon glue) packed with $8 \mathrm{mg}$ crystalline testosterone (Diosynth, Oss, The Netherlands). These were implanted subcutaneously in the neck region under local anesthesia with lidocaine (Xylocaine, Astra, Rijswijk, The Netherlands). The incision was closed with stitches.

We used hart puncture to draw blood from embryos and chicks younger than 4 days after hatching. Before puncture we anaesthetized these chicks and embryos with ether and afterward we killed them by exposing them to an overdose of ether. For older chicks we draw blood from the brachial wing vein and these chicks were kept alive. We collected $0.5-0.8 \mathrm{ml}$ blood with a heparin-rinsed needle and syringe within $5 \mathrm{~min}$ of capturing the bird (weight of the birds 200-300 g.). After centrifugation, we stored the plasma at $-20^{\circ} \mathrm{C}$ until hormonal analysis.

\section{Behavioral Tests}

This adult intruder test was a slight modification of the standard stimulus test that was developed for young black-headed gulls by Groothuis (1989b). During this test the observer was standing in front of the open door of the cage and handled the model. Each test consisted of five successive periods of $1 \mathrm{~min}$ duration: (1) the model was held in front of the open door of the cage; (2) the model was held in the middle of the cage (the chicks then often moved to the nest box at the back of the cage); (3) the model was held close to the birds and each bird was touched three times with the bill of the model; (4) as in (2); (5) as in (1). To avoid habituation, the model was kept continuously in motion by slowly moving the model in the horizontal plane and by gently shaking the model vertically. Behavioral observations were recorded on voice tape.

As a measurement of the responsiveness to an aggressive challenge, the frequency of the Oblique display was taken. This display is the most conspicuous and frequently performed aggressive display in blackheaded gull chicks and consists of an erect posture accompanied by a loud call (see Groothuis, 1989a, 1989b).

\section{Radioimmunoassay}

We measured testosterone concentrations by radioimmunoassay as described by Verjans, Cooke, de Jong, de Jong, and van der Molen (1973) with the modifications described by Dieleman, Kruip, Fontijne, and de Jong (1983). We used two different aliquots (range 5-200 $\mu \mathrm{l}$ ) to test for parallelity, which were extracted with n-hexane: diethylether $(4: 1, \mathrm{v} / \mathrm{v})$. The main cross-reactivities were $49.7 \%$ for $5 \alpha$-dihydrotestosterone, $7.64 \%$ for 4 -androstene- $3 \beta, 17 \beta$-diol, and $3.35 \%$ for androstenedione. The interassay coefficient of variation was $14 \%$, the intraassay coefficient $10 \%$. The limit of quantification was $0.05 \mathrm{ng}$ testosterone $/ \mathrm{ml}$ blood plasma. We used only those data of which the duplicate values differed less than $15 \%$.

\section{Statistical Treatment}

The data of levels of testosterone and of frequencies of Oblique showed skewed distributions. In order to apply parametrical tests (ANOVA and Students' $t$ tests) we logarithmically transformed the data in case of levels of testosterone and Poisson transformed them in case of Oblique frequencies (Zar, 1984). After transformation the data showed normal distributions and parametric tests were applied except in the case where the difference between control and challenge induced testosterone levels were compared. We averaged the data of those birds that were held in the same cage to avoid pseudo replication. This yielded a low sample size for the experimental group, which did not allow us to assess whether the data were normally distributed. Therefore we used the Mann-Whitney test in these cases. Two-tailed $P$ values were used with the threshold of significance set to alpha $=0.05$. 


\section{Dose Considerations}

In one of our earlier studies (Groothuis and Meeuwissen, 1992) many black-headed gull chicks (in contrast to juveniles), treated with testosterone for 4 weeks or longer developed impediment of breathing due to effects of testosterone on the syrinx, in contrast to juveniles treated in the same way. To find out how to reduce this harmful effect of testosterone treatment in chicks a pilot study was carried out. Eight chicks were implanted with testosterone filled tubes, each with a different tube length, for a period of only two weeks. The study showed: (1) a dose dependent effect of testosterone on aggressive behavior that seemed to level off at tube lengths between 10 and $15 \mathrm{~mm}$; (2) no harmful effects of testosterone within the first 10 days of implantation, the intended period of treatment in experiment 2 . Therefore, we chose to treat the chicks in experiment 2 with testosterone implants of $10 \mathrm{~mm}$ tube length for 10 days. Still, 4 of the 12 testosterone-treated birds died during the experiment. Although these birds died after removal of the implantation and only one of these appeared to have difficulties with breathing, the results strongly suggest that a 10 days treatment with testosterone, as used by us, has long-term detrimental effects on the chicks. This should be taken into account in designing future studies with this species. Mortality of all the untreated birds of experiment 1 and of the controls of experiment 2 was only $3 \%$. Subsequent experiments showed that a 10 -day hormonal treatment was much less harmful when given earlier in life, starting around day 6.

\section{RESULTS}

\section{Experiment 1: Effect of Social Challenges on Basal T Levels}

\section{Behavior and testosterone in the nonchallenged} condition. We never observed aggressive behavior between pseudo-siblings in the nonchallenged condition. Occasionally we tested the responsiveness of these chicks to an aggressive challenge by means of our stuffed model test. It appeared to be extremely difficult to elicit an aggressive response. The chicks mainly crawled together and hid from the introduced model of an adult bird. This behavior was very distinct from the behavior of chicks that were raised in larger groups and of several pseudo-families that were treated with testosterone (see below).

Levels of testosterone in the nonchallenged condition were highest in the period around hatching. After hatching the level of testosterone decreased twofold
(Fig. 1). A one-way ANOVA showed that this decrease over age is statistically significant $(F(3,29)=2.96$; $P<0.05)$. When the data over the first age class were left out of the ANOVA analysis no significant effect of age remained $(F(2,15)=0.33$; n.s.).

Behavior and testosterone in the challenged condition. We frequently observed the chicks to get an impression of the behavior of these birds in the large groups. These observations confirmed our expectations that during the first week after grouping in the challenged condition the chicks vigorously defended a territory against chicks of other pseudo-families. These territories usually consisted of some space around their nest boxes. In addition, the chicks appeared to be highly aggressive during encounters near the communal feeding and water trays. This aggression consisted mainly of display. Overt aggression only occurred when the victim was not at its own territory, and the pecked bird always escaped quickly back to its own place. In the course of the first and second week after the start of the experiment, territorial disputes became less frequent and the pseudofamilies avoided each other around the water and food tray. However, when we at this time induced a territorial conflict by introducing a model of an adult bird the chicks were still highly responsive to this stimulus with aggressive behavior.

The levels of testosterone changed in the course of the experiment in line with the change in spontaneous aggressive behavior. The two levels of the Large Group Challenge condition and the Territorial Challenge condition (the three data points of the "Territorial Challenge" condition were also taken together to avoid pseudo replication), were much higher than those of the twelve unchallenged groups of the same age (7 groups in age class 5-15, 5 in 15-25; MWU test: $U=0 ; P<0.05$; Fig. 1). After the first week since housing the chicks in large groups, levels of testosterone in the two groups of challenged chicks declined sharply to the levels of controls of the same age (5 groups in age class 15-25, 6 in 25-35; MWU test: $U=$ $4 ;$ n.s.). This decline was found for 14 of the 15 chicks of which we took paired samples (Paired $t$ test: $t(14)=8.61 ; P<0.001)$. Thus social stimulation, due to the grouping of unfamiliar birds induced a temporary increase in testosterone production.

\section{Experiment 2: Priming by Temporary $T$ Implantation}

Testosterone implants resulted in plasma levels of testosterone of $0.24 \pm 0.03 \mathrm{ng} / \mathrm{ml}$. This was signifi- 


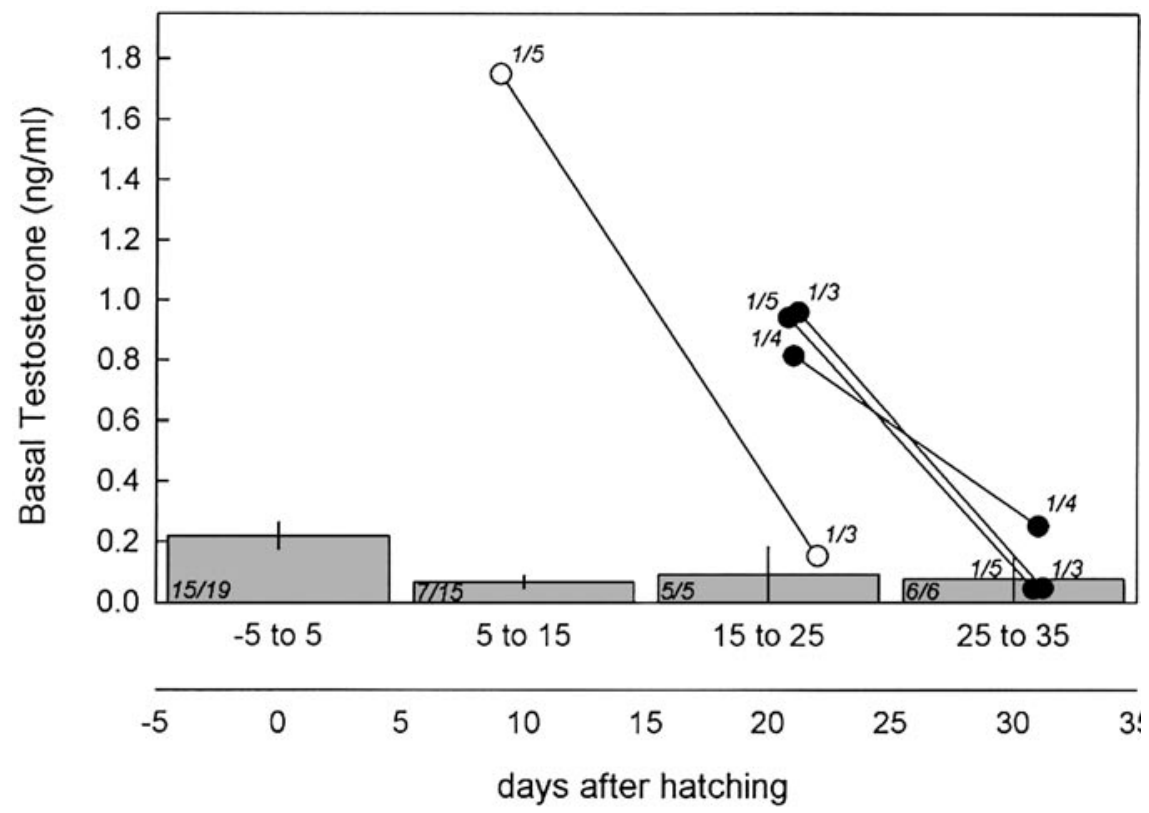

FIG. 1. Basal levels of testosterone in blood plasma of untreated chicks in the nonchallenged condition (upper $x$ axis; bars) and the challenged condition (lower x axis; white dots: Large Group Challenge condition; black dots: Territorial Challenge condition). Age is expressed in days after hatching. Data are mean ( \pm SEM) values per pseudo-family, except for chicks younger than 4 days of age, which could hardly have influenced each other. $N$ values are plotted in italic (statistical unit/total number of sampled chicks. Bars are based on data clustered in periods of 10 days.

cantly higher than basal levels of testosterone in the first week after removal of the implant: $0.07 \pm 0.04$ $\mathrm{ng} / \mathrm{ml}$ (paired $t(7)=5.61 ; P<0.001$ ). It was also higher than basal levels of testosterone of the $C$ group: $0.08 \pm 0.01 \mathrm{ng} / \mathrm{ml}(t(7)=3.68 ; P<0.01)$.

During testosterone treatment birds of the T group performed more Oblique display in response to the model tests than birds of the $C$ group of the same age $(t(11)=2.20 ; P<0.05)$ (Fig. 2, day 18). Three weeks after termination of testosterone treatment the frequency of the Oblique was still significantly higher in the $\mathrm{T}$ group than in the $\mathrm{C}$ group $(t(11)=3.23 ; P<$ 0.01) (Fig. 2, day 39). In the period after treatment basal levels of testosterone of both groups were, however, at a similar low level ( $\mathrm{T}$ respectively $\mathrm{C}$ birds, $0.07 \pm 0.04$, respectively $0.08 \pm 0.01 \mathrm{ng} / \mathrm{ml} ; t(11)=$ $1.27 ;$ N.S.).

At 39 days after hatching in the $\mathrm{T}$ group ( 3 weeks after the end of $\mathrm{T}$ treatment) the level of testosterone in reaction to an aggressive challenge was $0.46 \pm 0.12$ $\mathrm{ng} / \mathrm{ml}$. This was more than six times higher than the basal level of testosterone after the end of $\mathrm{T}$ treatment in this group $(0.07 \mathrm{ng} / \mathrm{ml}$, see above; paired $t(6)=$ 5.37; $P<0.01)$. Around this period in the control group, the level of testosterone immediately after challenging was $0.75 \pm 0.28 \mathrm{ng} / \mathrm{ml}$, which was more than nine times higher than their basal level of testosterone $(0.08 \mathrm{ng} / \mathrm{ml}$, see above; paired $t(4)=2.85 ; P<0.05)$. The levels of testosterone after challenging did not differ significantly between the $\mathrm{T}$ and $\mathrm{C}$ groups $(t(10)=0.38 ;$ N.S. $)$, despite the clear differences in the behavioral response.

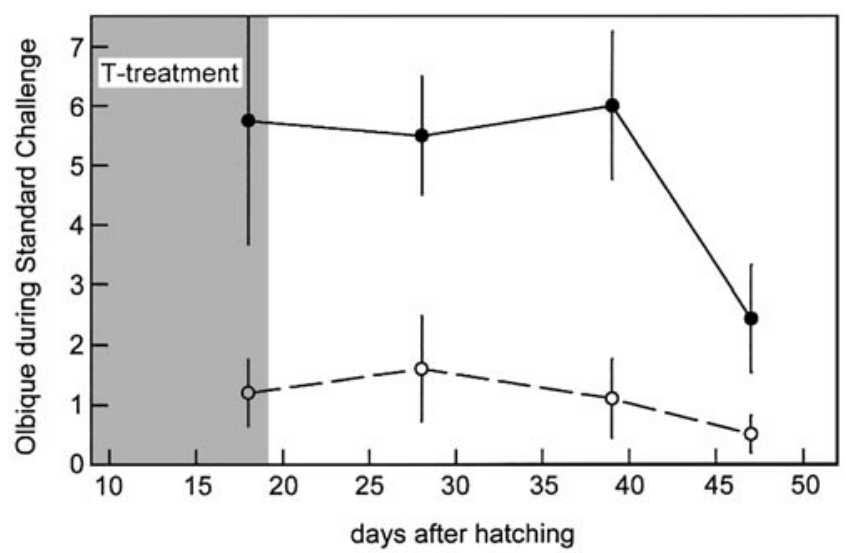

FIG. 2. Effect of testosterone treatment in black-headed gull chicks on the frequency of Oblique display during a standard aggressive challenge. Plotted are mean $( \pm$ SEM) values for testosterone-treated chicks (T group: closed dots) and sham-treated control chicks (C group: open dots). Testosterone tubes were implanted at 9 days and removed at 19 days after hatching (shaded area). 
a
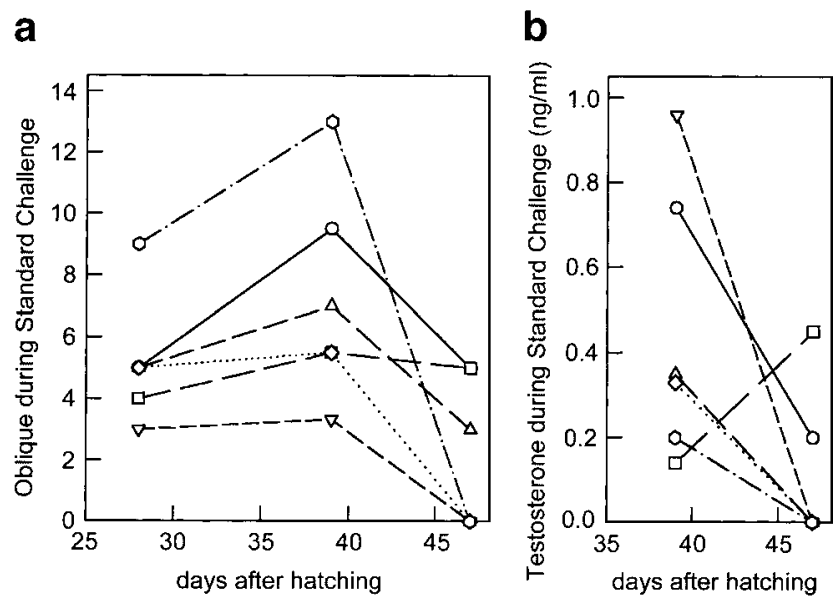

FIG. 3. Individual changes over age (days after hatching) in: (a) frequency of Oblique display, and (b) blood plasma levels of testosterone during an aggressive challenge, in black-headed gull chicks exposed to a 10-day treatment with testosterone between day 9 and day 19. Behavior was observed during a standard aggressive challenge. For measuring levels of testosterone during an aggressive challenge, blood was drawn within 5 min after termination of the standard aggressive challenge.

At 47 days after hatching (4 weeks after the end of T-treatment) the response to the aggressive challenge in the $\mathrm{T}$ group decreased (Birds of the T group, age 39 versus age 47 , paired $t(5)=2.82 ; P<0.05$ ) (Figs. 2 and $3 a)$. It was no longer significantly different from that of the C group (C group versus T group age 47, $t(11)=1.42$; n.s.) (Fig. 2). In four out of the six T-birds at this age the level of testosterone directly after the challenge was below the limit of quantification (Fig. $3 b)$. The only bird that did not show a strong decrease in Oblique display during this period was also the only bird that did not show a decrease in testosterone level during the aggressive challenge test (Figs. 3a and 3b: squared symbols).

\section{DISCUSSION}

\section{Evidence for the Challenge Hypothesis in Gull Chicks}

The main aim of this paper is to test the challenge hypothesis for the regulation of aggressive behavior early in life and outside the sexual context. Therefore we studied the relationship between testosterone, aggressive challenges, and territorial behavior in blackheaded gull chicks. We demonstrated that testosterone played an important role in facilitating this aggression and that the production of this hormone was under the influence of social challenges. Evidence for this is provided by the aggressive response to an aggressive challenge being much stronger in chicks treated with testosterone than in controls (see Groothuis and Meeuwissen, 1992). Further, untreated chicks forced to defend territories had elevated basal blood plasma levels of testosterone demonstrating a stimulating effect of a social challenge on $\mathrm{T}$ production. Finally, testosterone was rapidly, but temporarily, elevated when the chicks were aggressively challenged in a period when basal levels of the hormone were low. These results show the main premise of the challenge hypothesis for our birds.

It has been suggested that aggressive behavior is only under the influence of testosterone when it is performed in a sexual context, and that birds are only able to react quickly with a testosterone surge to a challenge when in reproductive condition (Schwabl, 1992; Wingfield and Monk, 1992; Wingfield, Jacobs, and Hillgarth, 1997). We collected our data from chicks that never showed any sign of sexual activity: they performed neither copulatory behavior nor sexual displays. Our results clearly show that aggression outside the sexual context can be under the influence of testosterone and that this hormone was produced by young chicks contingent on aggression. This may perhaps be explained by the finding that in young birds testosterone production can take place in the adrenals, an organ well known to produce fast surges of steroids in reaction to a wide variety of stressors (Tanabe, Yano, and Nakamura, 1983; Boswell, Hall, and Goldsmith, 1995).

\section{Priming Effects of Early Testosterone}

Chicks that were exposed to experimentally increased levels of testosterone showed persistently high levels of aggressive behavior in response to an aggressive challenge even after termination of treatment when basal levels of testosterone were low again. Groothuis and Meeuwissen (1992) found similar results for juvenile black-headed gulls. They interpreted this as a change in the causation of aggressive behavior by which in the course on ontogeny the behavior becomes independent of the hormone. We now could show that despite the low basal levels of testosterone the chicks still produce a brief elevation in testosterone plasma levels when they were aggressively challenged, indicating no such change.

The elevation in testosterone levels after a challenge was also found in untreated chicks. However, these birds showed relatively low levels of aggression dur- 
ing these challenges. This indicates that temporary exposure to testosterone increases the sensitivity of the aggressive response to subsequent acute elevations in testosterone levels induced by aggressive challenges. This suggests an organizing effect of testosterone in early development, sensitizing the central aggressive response pathways.

Interestingly we found temporarily high basal $\mathrm{T}$ levels in chicks around hatching. These initial levels may already play a role in the proposed sensitization of aggressive behavior. Such a priming effect might even occur much earlier in ontogeny. Schwabl (1993, 1996) showed in canaries that egg yolk contains maternal androgens that influence the competitive abilities of the chicks after hatching. Eggs of the blackheaded gull have been shown to contain high levels of androgens too (Groothuis and Schwabl, 2002; Eising, Eikenaar, Schwabl, and Groothuis, 2001) and the relatively high levels around hatching in a period that the chick is still using yolk may still be of maternal origin.

\section{Functional Consequences}

In the field chicks are frequently challenged by chicks of other families (Groothuis, 1989b). This situation was mimicked in our challenged conditions: the Large Group Challenge condition and the Territorial Challenge condition. In these conditions chicks showed elevated levels of testosterone similar to those resulting from our hormonal treatment in experiment 2 which induced the long term increase in sensitivity to the hormone. It is likely that this also occurs under field conditions where testosterone may be induced by early aggressive interactions leading to elevated levels of testosterone.

It has been proposed that the function of brief elevations in levels of $\mathrm{T}$ in response to an aggressive challenge is to enable a bird to perform aggressive behavior without being constantly exposed to high levels of testosterone. This is an important mechanism for adult birds in the period when parental care is provided because experimentally elevated $\mathrm{T}$ is known to inhibit parental behavior (for reviews see Wingfield et al., 1990; Ketterson et al., 1996). This can obviously not be the adaptive value of the mechanism in the black-headed gull chicks. We suggest that the mechanism in these chicks is adaptive because exposure to chronic high levels of testosterone has negative developmental effects. Early exposure to testosterone has been shown to suppress growth in young birds (chickens: Fennell and Scanes, 1992; gulls: Ros, 1999), to suppress begging (Groothuis and Meeuwissen, 1992) and to induce a less cryptic plumage (Ros, 1999) in young black-headed gulls. During the chick period, selection for rapid growth will thus favor mechanisms that fine-tune the level of testosterone exposure to the need to perform aggressive behavior. Peaks in testosterone production early in ontogeny, resulting from interactions with conspecifics that intrude the nest territory, gradually increase the sensitivity to testosterone, thereby increasingly facilitating the behavioral responsiveness of the chicks without the need for elevated basal levels of testosterone. The gradual increase in sensitivity suggests that organizing effects of the hormone may already affect the behavior in the chick phase. Whether this effect is restricted to a sensitive period and how long-lasting the effect is, has been part of a separate study.

The finding that previous exposure to the hormone may be important to establish the relationship between testosterone and behavior as postulated in the challenge hypothesis is interesting in a broader framework. First it may be applicable for adult animals too. In monogamous bird species, during the period in which the males are providing parental care, basal levels of testosterone are low but a close association exists between aggressive behavior and brief elevations in testosterone blood plasma levels during aggressive challenges (see Wingfield et al., 1990). The sensitivity of aggressive behavior to these peaks may be an effect of the high levels of testosterone to which these males are exposed in the beginning of the breeding season. Second, differences in the level of early social stimulation may lead to differences in early exposure to testosterone. This may be important for the development of individual differences within the same sex (see also van Oortmerssen, Dijk, and Schuurman, 1987; Moore, 1991; Clark and Galef, 1995).

\section{ACKNOWLEDGMENTS}

\footnotetext{
We are grateful to: Jaap Kruijt and Jaap Koolhaas for their help throughout the study, Jeroen Hazebroek for helping collecting the data, Ad van de Poll and Thea Blankenstein for doing the hormonal assays and Sjoerd Veenstra, Roelie Wiegman, and Tosca Boerée for their assistance in rearing the birds. This project was funded by Grant NR SLW-805.30.203 by SLW (Dutch Science Foundation). We thank two anonymous referees for suggestions that improved the manuscript.
}

\section{REFERENCES}

Boswell, T., Hall, M., and Goldsmith, A. R. (1995). Testosterone is secreted extragonadally by European quail maintained on short days. Physiol. Zoology 68(6), 967-984. 
Cash, K. J., and Evans, R. M. (1986). The occurrence, context and functional significance of aggressive begging behaviours in young American white pelicans. Behaviour 102, 119-128.

Clark, M. M., and Galef, B. G., Jr. (1995). Prenatal influences on reproductive life history strategies. Trends Ecol. Evol. 10, 151-153.

Dieleman, S. J., Kruip, T. A. M., Fontijne, P., de Jong, W. H. R., and van der Weyden, G. C. (1983). Changes in oestradiol, progesterone and testosterone concentrations in follicular fluid and in the micromorphology of preovulatory bovine follicles relative to the peak of luteinizing hormone. J. Endocrinol. 97, 31-42.

Drummond, H., and Osorno, J. L. (1992). Training siblings to be submissive losers: Dominance between booby nestlings. Anim. Behav. 44, 881-893.

Dufty, A. M., Jr. (1989). Testosterone and survival: A cost of aggressiveness? Horm. Behav. 23, 185-193.

Eising, C. M., Eikenaar, C., Schwabl, H., and Groothuis, T. G. G. (2001). Maternal androgens in black-headed gulls: Consequences for chick development. Proc. R. London B 268, 839-846.

Fennell, M. J., and Scanes, C. G. (1992). Inhibition of growth in chickens by testosterone, 5a-dihydrotestosterone and 19-nortestosterone. Poult. Sci. 71, 357-366.

Groothuis, T. (1989a). On the ontogeny of display behaviour in the black-headed gull: I. The gradual emergence of the adult forms. Behaviour 109, 76-123.

Groothuis, T. (1989b). On the ontogeny of display behaviour in the black-headed gull: II. Causal links between the development of aggression, fear and display behaviour: emancipation reconsidered. Behaviour 110, 161-204.

Groothuis, T., and Meeuwissen, G. (1992). The influence of testosterone on the development and fixation of the form of displays in two age classes of young black-headed gulls. Anim. Behav. 43, 189-208.

Groothuis, T. G. G., and Schwabl, H. (2002). Determinants of within and among clutch variation in yolk androgens in the blackheaded gull. Funct. Ecol. 16.

Harding, C. F. (1979). Hormone changes triggered by aggression in a natural population of blackbirds. Science 203, 918-920.

Hegner, R. E., and Wingfield, J. C. (1987). Social status and circulating levels of hormones in flocks of house sparrows, Passer domesticus. Ethology 76, 1-14.

Ketterson, E. D., Nolan, V., Jr., Cawthorn, M. J., Parker, P. G., and Ziegenfus, C. (1996). Phenotypic engineering: Using hormones to explore the mechanistic and functional bases of phenotypic variation in nature. Ibis 138, 70-86.

Mock, D. W., Lamey, T. C., Williams, C. F., and Pelletier, A. (1987). Flexibility in the development of heron sibling aggression: An intraspecific test of the prey-size hypothesis. Anim. Behav. 35, 1386-1393.

Moore, M. C. (1991). Application of organization-activation theory to alternative male reproductive strategies: A review. Horm. Behav. 25, 154-179.

Moss, T., Parr, R., and Lambin, X. (1994). Effects of testosterone on breeding density, breeding success and survival of red grouse. Proc. R. Soc. Lond. Ser. B Biol. Sci. 258, 175-180.
Nuñez De La Mora, A., Drummond, H., and Wingfield, J. C. (1996). Hormonal correlates of dominance and starvation-induced aggression in chicks of the blue-footed booby. Ethology 102, 748-761.

Paterson, I. J. (1965). Timing and spacing of broods in the blackheaded gull Larus ridibundus. Ibis 107, 433-459.

Ramenofsky, M. (1984). Endogenous plasma hormones and agonistic behavior in male Japanese quail, Coturnix coturnix. Anim. Behav. 32, 698-708.

Ramos-Fernandez, G., Nuñez de la Mora, A., Wingfield, J. C., and Drummond, H. (2000). Endocrine correlates of dominance in chicks of the blue-footed booby (Sula nebouxii): Testing the Challenge Hypothesis. Ethol. Ecol. Evol. 12, 27-34.

Ros, A. F. H. (1999). Effects of testosterone on growth, plumage pigmentation, and mortality in Black-headed Gull chicks. Ibis 141, 451-459.

Schwabl, H. (1992). Winter and breeding territorial behaviour and levels of reproductive hormones of migratory European robins. Ornis Scand. 23, 271-276.

Schwabl, H. (1993). Yolk is a source of maternal testosterone for developing birds. Proc. Natl Acad. Sci. USA 90, 11,446-11,450.

Schwabl, H. (1996). Maternal testosterone in the avian egg enhances postnatal growth. Comp. Biochem. Physiol. 114, 271-276.

Tanabe, Y., Yano, T., and Nakamura, T. (1983). Steroid synthesis and secretion by testes, ovary, and adrenals of embryonic and postembryonic ducks. Gen. Comp. Endocrin. 49, 144-153.

Terkel, A. S., Moore, C. L., and Beer, C. G. (1976). The effects of testosterone and estrogens on the rate of long-calling vocalization in juvenile laughing gulls, Larus atricilla. Horm. Behav. 7, 49-57.

van Oortmerssen, G. A., Dijk, D. J., and Schuurman, T. (1987). Studies on wild house mice. II. Testosterone and aggression. Horm. Behav. 21, 139-152.

Verjans, H. L., Cooke, B. A., de Jong, F. H., de Jong, C. M. M., and van der Molen, H. J. (1973). Evaluation of a radioimmunoassay for testosterone estimation. J. Steroid Biochem. 4, 665-676.

Wingfield, J. C. (1985). Short-term changes in plasma levels of hormones during establishment and defense of a breeding territory in male song sparrows, Melospiza melodia. Horm. Behav. 19, 174-187.

Wingfield, J. C., and Monk, D. (1992). Control and context of yearround territorial aggression in the non-migratory song sparrow Zonotrichia melodia morphna. Ornis Scand. 23, 298-303.

Wingfield, J. C., Newman, A. L., Hunt, G. L., and Farner, D. S. (1982). Endocrine aspects of female-female pairing in the Western Gull (Larus occidentalis wymani). Anim. Behav. 30, 9-22.

Wingfield, J. C., Hegner, R. E., Dufty, A. M., Jr., and Ball, G. F. (1990). The challenge hypothesis: Theoretical implications for patterns of testosterone secretion, mating systems, and breeding strategies. Am. Nat. 136, 829-846.

Wingfield, J. C., Jacobs, J., and Hillgarth, N. (1997). Ecological constraints and the evolution of hormone-behavior interrelationships. Ann. N. Y. Acad. Sci. 807, 22-41.

Zar, J. H. (1984). Biostatistical Analysis. Prentice-Hall, Englewood Cliffs, NJ. 